\title{
Stentgraftimplantation bei femoralen Aneurysmen mit Hilfe von kontinuierli- chem Gefäßultraschall
}

Die Hauptlokalisation peripherer arterieller Aneurysmen ist die A. poplitea. Sehr viel seltener ist die A.femoralis superficialis betroffen (Dimakokos PB et al., Vasc Med 1998; 3: 275), dann meist als Pseudoaneurysma nach vorausgegangener Bypass-Operation oder einer perkutanen aortalen Endograft-Implantation (Biederer J et al., Eur Radiol 1999; 9: 1678).

Zur Ausschaltung des Aneurysmas gibt es zwei kurative Ansätze: zum einen die Operation, zum anderen in zunehmendem Maße die Implantation eines Stentgrafts, also eines mit Dacrongewebe überzogenen Gefäßstents (Dorffner R et al., Fortschr Röntgenstr 1998; 168; 3: 275). Ein teilthrombosiertes Aneurysma lässt sich durch die i.a. DSA oft nicht in seiner ganzen Ausdehnung nachweisen. Dementsprechend schwierig ist die Planung und Durchführung der Stentimplantation allein aufgrund der DSA. Beim Gefäßultraschall hingegen ist das Schallfenster meist zu kurz, die ganze Länge des Aneurysmas kann nicht auf einer Aufnahme abgebildet werden. Erst in jüngster Zeit stehen Geräte zur Verfügung, die eine längere Strecke eines Gefäßes erfassen (Beissert $M$ et al., Radiologe 1998; 38: 410).

Wir berichten über zwei Patienten mit femoralen Aneurysmen, bei denen erstmalig unter kontinuierlicher sonographischer Kontrolle ein Stentgraft implantiert wurde.

\section{Fallbeschreibung}

Fall 1

Ein 72-jähriger männlicher Patient klagte seit über 4 Monaten über eine deutlich an Größe zunehmende pulsierende Resistenz am linken Oberschenkel. Drei Jahre zuvor war eine Thrombektomie aus einem femorokruralem Bypass durchgeführt worden. Seitdem war der Patient beschwerdefrei. Als Vorerkrankungen waren eine arterielle Hypertonie, eine linksventrikuläre kardiale Dekompensation bei koronarer Herzkrankheit, eine chronische Niereninsuffizienz im
Stadium der kompensierten Retention und ein Diabetes mellitus Typ II bekannt. Somit galt es für die Diagnostik und Interventionsplanung möglichst Kontrastmittel zu sparen oder zu vermeiden.

Mit Hilfe des Gefäßultraschalls wurde ein ausgedehntes teilthrombosiertes femorales Aneurysma, vom mittleren Anteil des femorocruralen Bypasses bis zum Adduktorenkanal reichend, diagnostiziert. Die Längsausdehnung betrug $9 \mathrm{~cm}$, der Querdurchmesser maximal $5,8 \mathrm{~cm}$, der Thrombus war bis $4 \mathrm{~cm}$ breit. Durch eine kontinuierliche Bildgebung mit Flussdokumentation wurde sonographisch gezielt nach transfemoraler Punktion eine Stentimplantation vorgenommen. Nach Fixieren des Stentgraft am kranialen und kaudalen Ende wurde das Aneurysma ausgeschaltet. Der Patient ist seitdem beschwerdefrei, ein Pulsieren am Oberschenkel wurde nicht mehr bemerkt. Kontrollen ergaben keine Reperfusion des Aneurysmas.

\section{Fall 2}

Ein 73-jähriger männlicher Patient klagte seit einem halben Jahr über eine tastbar pulsierende, an Größe zunehmende Resistenz am rechten Oberschenkel. 6 Jahre zuvor war eine Thrombektomie der rechten A.femoralis superficialis durchgeführt worden. An Vorerkrankungen waren eine arterielle Hypertonie, eine Mitralinsuffizienz und eine Hyperlipoproteinämie bekannt. Die zwei Monate zuvor durchgeführte intraarterielle DSA zeigte lediglich eine generelle Gefäßektasie der A.femoralis superficialis.

Durch Gefäßultraschall wurde ein ausgedehntes teilthrombosiertes femorales Aneurysma diagnostiziert. Die Längsausdehnung betrug $8,5 \mathrm{~cm}$, der Querdurchmesser maximal $5,4 \mathrm{~cm}$, der Thrombus war bis $\mathrm{zu} 3,5 \mathrm{~cm}$ breit. Mit Hilfe der kontinuierlichen sonographischen Bildgebung mit Flußdokumentation wurde nach transfemoraler Punktion ein Stentgraft zur Ausschaltung des Aneurysmas eingebracht. Kontrollen konnten eine Reperfusion des femoralen
Aneurysmas ausschließen, das Pulsieren trat nicht mehr auf.

\section{Diskussion}

Über perkutane transluminäre Dilatationen bei Gefäßstenosen, die unter Ultraschallkontrolle behoben wurden, ist bereits in der Literatur berichtet worden (Katzenschlager R et al. Int Angiol 1998; 17: 80). Über eine periphere Stentgraftimplantation bei Gefäßaneurysmen mit Hilfe von Ultraschall liegen unseres Wissens noch keine Mitteilungen vor.

Um die teilthrombosierten Aneurysmen durch einen Stentgraft ausschalten zu können, bedurfte es eines bildgebenden Verfahrens, das die exakte Lokalisation, Ausdehnung und Perfusion des Aneurysmas erfasst und gleichzeitig den thrombosierten Anteil darstellt. Wir verwendeten ein Gerät mit einem 7,5-MHzSchallkopf mit kontinuierlicher Flussdokumentation im Power Mode (ColorSieScape, Firma Siemens, Erlangen), das „Panorama“-Bilder der Gefäße im RealTime-Bild bis zu $60 \mathrm{~cm}$ Längsausdehnung erfasst. Dabei wertet der ImageProzessor die Ähnlichkeit aufeinanderfolgender Einzelbilder aus, bestimmt die relative Position des Schallkopfes, richtet die Bilder aus und fügt sie zu einem Gesamtbild zusammen. Der zusätzliche Power-Mode erlaubt es in der kontinuierlichen Darstellung, das durchflossene Lumen gegenüber dem Thrombus abzugrenzen.

Bei einer Längsausdehnung des femoralen Aneurysmas von $9 \mathrm{~cm}$ gelingt die vollständige Untersuchung einschließlich einer Dokumentation der Messparameter nur in der Panoramabildgebung (Abb. 1 und 2). Der Gefäßultraschall ist der Angiographie in Bezug auf die Beurteilung der Wand- und Umgebungsstrukturen überlegen. Die DSA zeigte bei ausgeprägter Thrombusbildung in den femoralen Aneurysmen beider Patienten nur unregelmäßige Gefäßkonturen.

Um sich die Vorteile einer sonographischen Flussdokumentation zunutze zu machen und Kontrastmittel zu sparen, wurde in beiden Fällen die transfemorale angiographische Stentimplantation sonographisch gezielt durchgeführt (Abb.3). Über den gesamten ektatisch und aneurysmatisch erweiterten Gefäßabschnitt konnte dabei auch im Panoramabildverfahren in Real-Time der Be- 

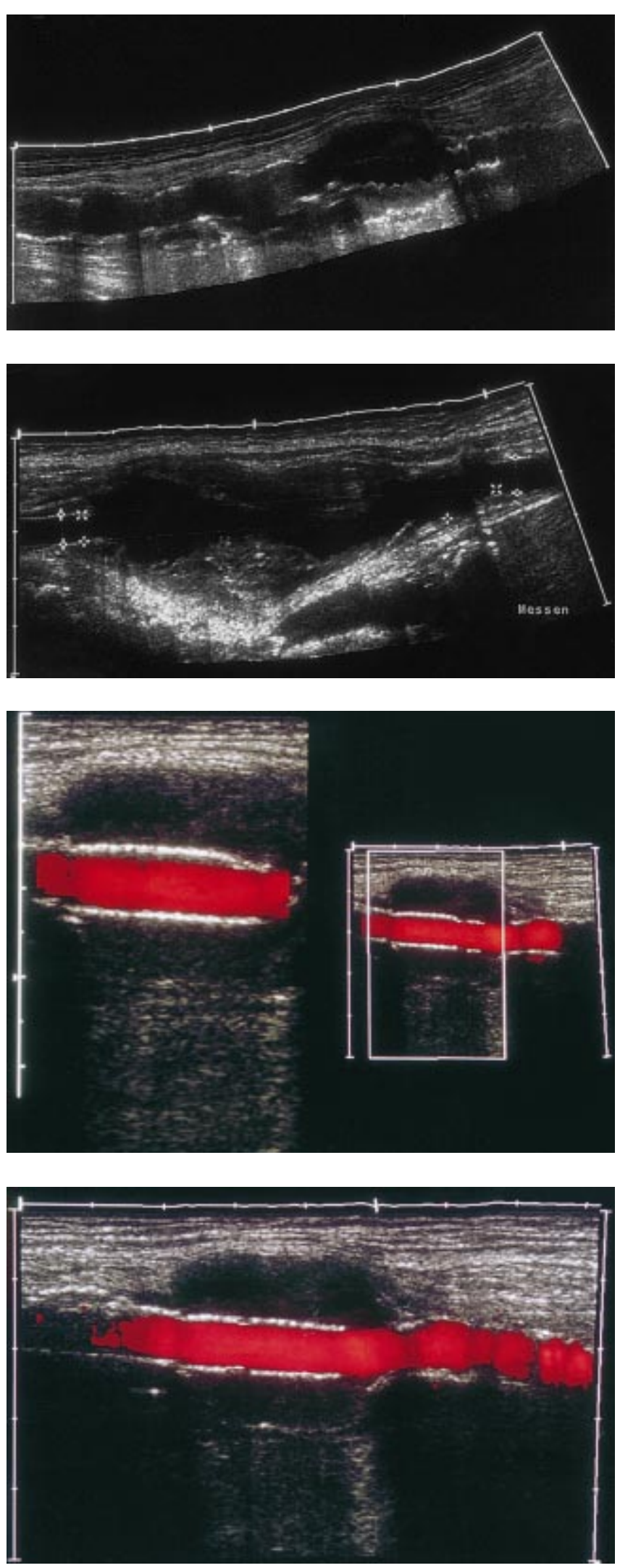

Abb. 1 Kontinuierliche Bilddokumentation eines femoralen Aneurysmas bei genereller Gefäßektasie und Z. n. Thrombektomie nach Anlage eines femoro-cruralen Bypasses.

Abb. 2 Kontinuierliche Bilddokumentation eines $9 \mathrm{~cm}$ langen, bis $6 \mathrm{~cm}$ breiten teilthrombosierten femoralen Aneurysmas. Messdokumentation zur Stentimplantation.

Abb. 3 Stentgraft Implantation (Hemobahn). Dokumentation mit kontinuierlichem PowerMode. Aneurysma vollständig ausgeschaltet. Real-TimeBildgebung.

Abb. 4 AbschlieBende Dokumentation nach Stentgraft Implantation (Power-SieScape). reich des Aneurysma dargestellt werden, in dem der Endograft fixiert wurde. Der Schallkopf wurde freihändig geführt ohne Positionssensoren oder Führungsarme. Mit Hilfe eines gecoverten Stentgrafts von $10 \mathrm{~cm}$ Länge und $7 \mathrm{bzw} .10 \mathrm{~mm}$ Breite (Hemobahn, Fa. Gore) wurde eine vollständige Ausschaltung der Aneurysmen erreicht (Abb. 4).

Es ist mit Hilfe des Gefäßultraschalls eine zeitsparende exakte Verankerung und gezielte Dilatation des Stentgrafts möglich, der sich im Ultraschall gut abgrenzen lässt. Eine Kontrolle durch endovasalen Ultraschall erübrigt sich. Lediglich zum Ausschluss peripherer Embolien wurden noch geringe Kontrastmittelmengen für die i.a. DSA benötigt. Dies ist ein entscheidender Vorteil für die Interventionen bei Patienten mit kompensierter Niereninsuffizienz.

Der Einsatz neuer sonographischer Untersuchungstechniken in der Interventionsplanung, Durchführung und Kontrolle eröffnet für die Zukunft neue Möglichkeiten, insbesondere in Bezug auf eine Kontrastmittel- und Strahlendosisreduktion.

E. M. Jung, Klinikum Passau, W. Ritter, I. Bär, Klinikum Nürnberg Süd 